The FASEB Journal express article 10.1096/fj.03-1338fje. Published online October 29, 2004.

\title{
Differential insertion of GPI-anchored GFPs into lipid rafts of live cells
}

\author{
Daniel F. Legler, ${ }^{*}{ }^{\dagger}$ Marie-Agnès Doucey, ${ }^{\dagger}$ Pascal Schneider, ${ }^{\dagger}$ Laurence Chapatte, $^{\dagger}$ \\ Florent C. Bender, ${ }^{\dagger}$ and Claude Bron $^{\dagger}$
}

*Department of Biology, Division of Immunology, University of Konstanz, D-78457 Konstanz, Germany; and ${ }^{\dagger}$ Institute of Biochemistry, University of Lausanne, BIL Biomedical Research Center, CH-1066 Epalinges, Switzerland

Corresponding author: Daniel F. Legler, Ph.D., Department of Biology, Division of Immunology, University of Konstanz, Universitätsstrasse 10, Room P1105, D-78457 Konstanz, Germany. E-mail: Daniel.Legler@uni-konstanz.de

\section{ABSTRACT}

Partitioning of proteins in cholesterol and sphingolipid enriched plasma membrane microdomains, called lipid rafts, is critical for many signal transduction and protein sorting events. Although raft partitioning of many signaling molecules remains to be determined, glycosylphosphatidyl-inositol (GPI)-anchored proteins possess high affinity for lipid rafts and are currently exploited as markers to investigate fundamental mechanisms in protein sorting and signal transduction events. In this study, we demonstrate that two recombinant GPI-anchored green fluorescent proteins (GFP-GPIs) that differ in their GPI signal sequence confer distinct localization in plasma membrane microdomains. GFP fused to the GPI signal of the decay accelerating factor GFP-GPI(DAF) partitioned exclusively in lipid rafts, whereas GFP fused to the GPI signal of TRAIL-R3, GFP-GPI(TRAIL-R3), associated only minimally with microdomains. In addition, we investigated the unique ability of purified GFP-GPIs to insert into membrane microdomains of primary lymphocytes. This cell surface painting allows rapid, stable, and functional association of the GPI-anchored proteins with the target cell plasma membrane. The distinct membrane localization of the two GFP-GPIs was observed irrespective of whether the GPI-anchored molecules were painted or transfected. Furthermore, we show that painted GFP-GPI(DAF) was totally dependent on the GPI anchor and that the membrane insertion was increased by the addition of raft-associated lipids such as cholesterol, sphingomyelin, and dipalmitoyl-phosphatidylethanolamine. Thus, this study provides evidence that different GPI signal sequences lead to distinct membrane microdomain localization and that painted GFPGPI(DAF) serves as an excellent fluorescent marker for lipid rafts in live cells.

Key words: glycosylphosphatidyl-inositol • green fluorescent protein

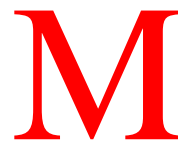

embrane microdomains, also called lipid rafts, arise from the preferential packing of sphingolipids and cholesterol in membrane subdomains, and they move within the fluid membrane phospholipid matrix. These microdomains are characterized by their 
discrete size, the liquid-ordered phase of their lipid components, and their light buoyant density. These features contribute to the lateral organization of the membrane, render microdomains resistant to solubilization with nonionic detergents, and allow their isolation by sucrose gradient centrifugation (1-6). Evidence that lipid rafts exist in live cells has been provided by fluorescence resonance energy transfer (FRET) experiments (7-9). With the use of this technique, domains of $<70 \mathrm{~nm}$ in diameter have been identified and can be disrupted by removal of cellular cholesterol.

Compartmentalization of the plasma membrane is of particular interest because it dictates the aggregation and segregation of proteins involved in signal transduction. Certain proteins preferentially partition into lipid rafts, in particular a group of functionally diverse cell surface proteins anchored in the outer leaflet of these membrane microdomains by a glycosylphosphatidyl-inositol (GPI) moiety. In addition, doubly acylated tyrosine kinases of the Src family, as well as a number of other peripheral signaling molecules bound to the inner leaflet of the membrane, also partition into lipid rafts $(1-3,5,10,11)$. Recently, the role played by lipid rafts in the sorting of lipids and proteins along both the secretory and endocytic pathways has been addressed (12-17).

Despite accumulating evidence suggesting the existence of lipid rafts in vivo, their size and composition under different physiological conditions remain to be clarified. To further our understanding of these domains, lipid-modified green fluorescent protein (GFP) variants have been generated and these partition to microdomains in live cells (9), thus providing a fluorescent marker of lipid rafts. Due to their preferential localization in lipid rafts, GPI-anchored GFP fusion proteins have also been used as markers to examine the dynamics of rafts and raftassociated proteins $(12,18-20)$. The demonstration that different naturally GPI-anchored proteins such as Thy- 1 and PrP are organized in different membrane domains on neuronal cell surfaces (21) highlights the critical importance of selecting the right GPI anchor for the design of a GFP-GPI fluorescent marker of lipid rafts.

After their extraction and purification, GPI-anchored proteins form micelles in solution and consequently can be re-incorporated into the plasma membrane when added to cells (reviewed in ref 22). Such protein transfer or "painting" has been successfully exploited to insert GPIanchored fusion proteins into plasma membranes where these proteins retained their natural function (22-28). Painting lipid rafts of cells with an appropriate GFP-GPI fusion protein should provide a useful tool for the study of microdomains in live cells and for investigating interactions between signaling molecules within the plasma membrane.

In this report, we describe two different GPI-anchored GFPs with distinct partitioning properties in the plasma membrane. In addition, we investigate the painting of lipid rafts of primary lymphocytes with fluorescent GFP-GPIs.

\section{MATERIALS AND METHODS}

\section{Cells}

Human peripheral blood lymphocytes (PBL) were isolated from donor blood buffy coats by separation on Ficoll-Paque followed by two steps of plastic adherence depletion of monocytes. 
PBL and the murine thymoma EL-4 cell line were cultured in RPMI 1640 supplemented with $10 \%$ FCS, $5 \mu \mathrm{g} / \mathrm{ml}$ penicillin, $5 \mu \mathrm{g} / \mathrm{ml}$ streptomycin, and $10 \mu \mathrm{g} / \mathrm{ml}$ neomycin (all from GibcoBRL, Life Technologies Inc., Paisley, GB). The human embryonic kidney cell line HEK293 was grown in DMEM supplemented with 10\% FCS and antibiotics.

\section{Antibodies}

Anti-epidermal growth factor receptor (EGFR) pAb was from Upstate Biotechnology (Lake Placid, NY), and HRP conjugated cholera toxin subunit B and anti-Flag mAb (M2) were from Sigma (Buchs, Switzerland). Anti-CD3 $\varepsilon$ pAb (M20) and anti-Fyn pAb (FYN3) were from Santa Cruz Biotechnology (Santa Cruz, CA). Anti-Thy-1 R287 antiserum was obtained by immunization with purified Thy-1 (29). Anti-GFP pAb was kindly provided by Dr. Viesturs Simanis (ISREC, Epalinges, Switzerland).

\section{Cloning of GPI-anchored GFPs}

The DNA fragment coding for the GPI signal of the TRAIL receptor 3 (TRAIL-R3; amino-acids 157-259), which also included multiple threonine, alanine, proline, and glutamine-rich repeats (TAPE repeats) (30), was cloned into the SalI/NotI-sites of the vector pKozak-HA (5' Kozak sequence in front of the leader sequence of haemagglutinin) derived from pCR3 (Invitrogen, San Diego, CA). The cDNA encoding the enhanced green fluorescent protein (GFP) was amplified by PCR using the primers 5'-AATCTCGAGGTGAGCAAGG and 5'-TAAGTCGACCTTGTACAGCTCGTCC (restriction sites are underlined) and the pEGFP-C1 (Clontech, Palo Alto, CA) as template. The PCR product was digested, purified, and subcloned into the Xhol/SalI-sites of pGPI(TRAIL-R3), resulting in the pGFPGPI(TRAIL-R3).

The cDNA fragment encoding the GPI signal of the decay-accelerating factor (DAF) was generated as follows. First, two oligonucleotides for the Flag epitope (5'CTCGAGTGCTAGCGACTACAAGGACGACGATGACAAGT and 5'TCTAGACTTGTCATCGTCGTCCTTGTAGTCGCTAGCAC) were annealed, digested, and cloned into the XhoI/XbaI-sites of pcDNA3 (Invitrogen). The GPI signal from DAF (aminoacids 345-381) was amplified by PCR using the primers 5'ATTATTCTAGACCAAATAAAGGAAGTGGAACC and 5'TAATTGGGCCCTAAGTCAGCAAGCCCATG and HUVEC cDNA. The amplified DNA was purified, digested, and subcloned into the XbaI/ApaI sites of pcDNA3-Flag. The SalI/NotIfragment containing the TRAIL-R3 GPI signal of pGFP-GPI(TRAIL-R3) was removed and replaced with the cDNA encoding the Flag-GPI(DAF) that has been amplified by PCR using the primers 5'-ATAGTCGACGACTACAAGGACGAC and ATTGCGGCCGCCTAAGTCAGCAAGC, resulting in the plasmid pGFP-GPI(DAF).

\section{Transfection of GFP-GPIs}

HEK293 cells were stably transfected in $10 \mathrm{~cm}$ dishes by the calcium phosphate procedure (31) for $8 \mathrm{~h}$ with $10 \mu \mathrm{g}$ of linearized plasmid cDNA. Cell clones were established by limiting dilution in the presence of $0.8 \mathrm{mg} / \mathrm{ml}$ of G-418 (Gibco-BRL, Life Technologies Inc.). The expression levels of the GPI-linked constructs were analyzed by flowcytometry using a FACStar (BectonDickinson, Erembodegen, Belgium). 


\section{Phosphatidylinositol-specific phospholipase C treatment of GFP-GPIs}

Viable cell suspensions were treated with phosphatidylinositol-specific phospholipase C (PIPLC) as described previously (32). Briefly, HEK293 cells $\left(2 \times 10^{5}\right)$ expressing GFP-GPI(DAF) or GFP-GPI(TRAIL-R3) were washed and resuspended in $100 \mu$ l of DME medium supplemented with $10 \%$ FCS and incubated for $2 \mathrm{~h}$ at $37^{\circ} \mathrm{C}$ with increasing concentrations (0-0.005 units) of PI-PLC from Bacillus thuringiensis (Glyco Inc., Navato, CA) before FACS analysis.

The GPI anchor of purified GFP-GPI(DAF) was cleaved by PI-PLC in serum free DMEM for 90 min at $37^{\circ} \mathrm{C}$, and the enzyme activity was blocked with $2 \mathrm{mM}$ EDTA as described previously (33).

\section{Isolation of lipid rafts}

HEK293 transfectants from two $15 \mathrm{~cm}$ culture dishes expressing GFP-GPI(DAF) or GFPGPI(TRAIL-R3) or $10^{8}$ painted lymphocytes were lysed on ice for $20 \mathrm{~min}$ in $2 \mathrm{ml}$ of $1 \%$ Triton X-100 in MNE buffer (25 mM MES, pH 6.5, $150 \mathrm{mM} \mathrm{NaCl,} 2 \mathrm{mM}$ EDTA) and homogenized (10 strokes) with a loose-fitting dounce homogenizer. The homogenates were mixed with $2 \mathrm{ml}$ of $90 \%$ sucrose prepared in MNE buffer and placed on the bottom of a centrifuge tube. The samples were then overlayed with $4 \mathrm{ml}$ of $35 \%$ sucrose and $4 \mathrm{ml}$ of 5\% sucrose in MNE buffer and centrifugated at $175,000 \mathrm{~g}$ in a Centrikon T-2070 centrifuge (Kontron Analytics, Zürich, Switzerland) for $20 \mathrm{~h}$. Fractions $(1 \mathrm{ml})$ were collected from the top of the gradient and subjected to Western blot analysis. The pellet present at the bottom of the gradient was sonicated in $1 \mathrm{ml}$ of MNE containing 1\% Triton X-100.

Labeling of HEK293 cells with ${ }^{14} \mathrm{C}$ cholesterol was performed as described previously (34, 35). Briefly, DME medium was incubated for $12 \mathrm{~h}$ with $0.4 \mu \mathrm{Ci}$ of $\left[{ }^{14} \mathrm{C}\right]$ cholesterol $(51 \mathrm{mCi} / \mathrm{mmol}$, Du Pont NEN, Boston, MA) and incubated with the cells for 48 h. The cells were then washed, cultured for $24 \mathrm{~h}$, and subjected to sucrose gradient centrifugation to isolate lipid rafts, and the $\left[{ }^{14} \mathrm{C}\right]$ cholesterol content in each fraction was measured.

\section{Purification of GFP-GPI fusion proteins}

HEK293 cells expressing recombinant GFP-GPI(DAF) or GFP-GPI(TRAIL-R3) were collected and washed twice with PBS. The GPI-linked GFPs were enriched by Triton X-114 phase extraction (36). The detergent phase contained $67-80 \%$ of the initial fluorescence, and the fluorescence intensity per milligram of protein increased by 18 -fold. The Triton X-114 phase was resuspended in $20 \mathrm{mM}$ Tris- $\mathrm{HCl}, \mathrm{pH}$ 8.0, containing 1\% Triton $\mathrm{X}-100$, and the lysate was applied to a mono Q HR5/5 column (Pharmacia, Uppsala, Sweden). The column was washed with $20 \mathrm{mM}$ Tris-HCl, $\mathrm{pH}$ 8.0, and Triton was exchanged with Brji96 by injecting $1 \mathrm{ml}$ of 1\% Brji96 in $20 \mathrm{mM}$ Tris-HCl, pH 8.0. After being washed extensively with Tris buffer containing $120 \mathrm{mM} \mathrm{NaCl}$, the GFP-GPI fusion proteins were recovered by a step elution using $0.4 \mathrm{M} \mathrm{NaCl}$ in Tris buffer and analyzed by SDS-PAGE followed by Western blotting or silver staining. The fluorescence intensity per milligram of protein after Triton X-100 extraction and mono Q purification increased by 50 -fold. 


\section{Cell surface painting of GFP-GPI fusion proteins}

For cell surface painting with GPI-linked GFP proteins, target cells were washed twice, resuspended in serum-free medium at 5-10 $\times 10^{6}$ cells $/ \mathrm{ml}$, and incubated with purified proteins $(0.5-5 \mu \mathrm{g} / \mathrm{ml})$ for $2 \mathrm{~h}$ or $36 \mathrm{~h}$ at $37^{\circ} \mathrm{C}$ with occasional inversion. Painted cells were extensively washed, and the membrane insertion efficiency assessed by FACS analysis and by confocal microscopy.

\section{Confocal laser scanning microscopy}

HEK293 cells expressing GFP-GPI fusion proteins were cultured for $24 \mathrm{~h}$ on chambered borosilicate coverglasses (Lab-TekII system, Nalge Nunc International, Naperville, IL). Painted EL-4 cells and human PBL were washed and placed on chambered coverglasses for 10 min. Live cells were immediately analyzed by confocal microscopy on an Axiovert 100 microscope (LSM510, Carl Zeiss, Jena, Germany) with a $\times 63$ oil objective. To detect the GFP, an argon laser was filtered at $488 \mathrm{~nm}$. Each image was the average of eight scans. Digital images were prepared using Adobe Photoshop ${ }^{\circledR}$.

\section{RESULTS}

\section{GFP-GPIs: fluorescent markers for lipid rafts}

GPI-mediated anchoring or palmitoylation and/or myristoylation of proteins are biological modifications that allow the association of proteins with the inner and outer leaflet of plasma membrane microdomains. We decided to exploit this natural process to generate an extracellular fluorescent marker for lipid rafts. We constructed two GFP-GPIs by fusing the GPI anchor addition sequence of DAF or TRAIL-R3 to an enhanced GFP variant. Both GFP constructs were stably transfected in HEK293 cells and revealed comparable protein expression levels as evidenced by FACS analysis (Fig. 1A). Confocal laser microscopy demonstrated that both GFPGPI fusion proteins localized at the plasma membrane (Fig. 1C). Only marginal intracellular staining was observed suggesting limited recycling of both membrane-associated proteins. To further characterize the two recombinant proteins, transfected cells were treated with PI-PLC, which cleaves specifically the ectodomains of many GPI-anchored proteins (32). After this treatment, 91\% of surface expressed GFP-GPI(DAF) was lost (Fig. 1A). In contrast, PI-PLC treatment released only 19\% of GFP-GPI(TRAIL-R3) from the cell surface of HEK293 transfectants. To investigate whether a higher concentration of PI-PLC could cleave GFPGPI(TRAIL-R3) more efficiently, we incubated transfected cells with increasing concentrations of PI-PLC. However, GFP-GPI(TRAIL-R3) remained rather PI-PLC insensitive even at high PIPLC concentrations, whereas GFP-GPI(DAF) cleavage by PI-PLC was concentration dependant (Fig. 1B). Native GPI-anchored TRAIL-R3 was also resistant to PI-PLC either when expressed at the cell surface or when purified from these cells (data not shown). TRAIL-R3 immunoprecipitated from metabolically labeled cells incorporated $\left[{ }^{3} \mathrm{H}\right]$ inositol, confirming that TRAIL-R3 is indeed a GPI-anchored protein (data not shown).

\section{Distinct localization of GFP-GPIs in lipid rafts}

To investigate the localization of GFP-GPI fusion proteins within the plasma membrane, HEK293 cells stably transfected with either GFP-GPI(DAF) or GFP-GPI(TRAIL-R3) were lysed 
in 1\% Triton X-100 and fractionated on a sucrose density gradient. As expected, both GPIanchored proteins were floated in the low-density fractions of the gradient, which contain the detergent-insoluble lipid rafts (Fig. 2). These fractions were enriched in cholesterol and contained the doubly acylated Fyn kinase as well as the raft-associated ganglioside GM1 (Fig. 2), whereas the epidermal growth factor receptor (EGFR) was excluded from microdomains. Interestingly, a remarkable difference in the lipid raft partitioning was observed for the two GPIfusion proteins: whereas only 25-55\% of the GFP anchored via the TRAIL-R3 GPI moiety was found in the cholesterol/sphingolipid-enriched light-density fractions, the GFP linked to the DAF GPI anchor exclusively partitioned in the lipid raft fractions. The same distribution of the GFPGPIs was observed when the fluorescence derived from the GFP was measured in each fraction of the sucrose gradient directly, rather than monitoring denatured protein by Western blotting. Wild-type TRAIL-R3 has been shown to contain 5 repeat units of 15 amino acids (TAPE repeats) located at the extracellular domain adjacent to the membrane docking site (30). We investigated whether these TAPE repeats play any role in lipid raft localization and PI-PLC accessibility. By comparing mutant TRAIL-R3 molecules having no (via deletion of amino acid 160-230), or only one (via deletion of amino acid 171-230), with TRAIL-R3 containing all five TAPE repeats (30), we observed the same degree of association with lipid rafts (Fig. 3A), indicating that the TAPE repeats have no influence on lipid raft localization. Furthermore, all three forms were insensitive to PI-PLC cleavage (data not shown).

To further investigate the different GFP-GPI proteins, we cloned the GPI addition sequence of TRAIL-R3 into the GFP-GPI(DAF) backbone vector by replacing DAF 345-381 with TRAIL-R3 $_{213-}$ 259. In the mature form of this GFP-TRAIL-R3 ${ }_{213-259}$, the GPI anchor will be added to Alanine 221 and the remaining amino acids (TRAIL-R3 $222-259$ ) will be cleaved off. The GPI anchor to the mature GFP-GPI(DAF) will be added to Serine 353 . Thus, the mature forms of GFP-TRAIL-R3 ${ }_{213-}$ 259 and GFP-DAF $345-381$ differ only in nine amino acids preceding the GPI addition sequence (Fig. 3B). We transfected these constructs in HEK293 cells and investigated their lipid raft localization. GFP-TRAIL-R3 $213-259$ still associated only partially with microdomains, whereas GFP-DAF ${ }_{345-381}$ exclusively partitioned in lipid rafts (Fig. 3B), providing evidence that the last nine amino acids of the mature protein or the GPI anchor itself is responsible for the distinct membrane localization of the two GFP-GPI molecules.

\section{Cell surface painting of T lymphocytes with purified GFP-GPIs}

One interesting property of purified GPI-anchored proteins is their ability to re-insert into the plasma membrane upon incubation with a target cell. To exploit this property for the fluorescent labeling of lipid rafts, we first enriched both GPI-anchored GFPs from HEK293-transfected cells by extraction in Triton X-114, a detergent known to disperse membrane proteins (36). GFP-GPIs were then further purified by ion-exchange chromatography. For cell surface painting, the murine EL-4 thymoma cell line and primary human PBL were incubated with purified GPIanchored GFPs at $37^{\circ} \mathrm{C}$. After $1.5-3 \mathrm{~h}$, cells were extensively washed and the plasma membrane insertion of painted proteins was analyzed by FACS (Fig. 4A). The efficiency of membrane insertion of the purified molecules was similar on EL-4 cells and PBL. Additionally, the expression level of GFP-GPIs on painted lymphocytes and transfected HEK293 cells was comparable. 
Faint cell-surface staining was observed after just a few minutes incubation, and this reached a plateau after $90 \mathrm{~min}$. Prolonged incubation, up to $6 \mathrm{~h}$, did not increase the efficiency of the surface labeling (data not shown). Painting of GFP-GPIs was temperature dependent since no insertion was observed at $4^{\circ} \mathrm{C}$, and painting was greatly reduced at room temperature (data not shown), confirming painting characteristics of other GPI-anchored proteins (22, 28, 37). Monitoring of painted cells by confocal microscopy clearly showed restricted localization of both GFP-GPIs at the plasma membrane (Fig. 4B). No intracellular labeling was observed, indicating that both proteins were retained at the cell surface and were not internalized. Painted molecules remained stably associated with the cell surface for up to $12 \mathrm{~h}$ followed by a progressive decrease in surface expression upon successive cell division (data not shown).

Finally, identical results were obtained with a number of different target cells, including primary murine $\mathrm{T}$ and B lymphocytes and human cell lines such as Jurkat $\mathrm{T}$ cells, EBV transformed BJAB and Raji B cells, and leukemia K-562 cells (data not shown).

\section{Role of the GPI anchor and carrier lipids for cell surface painting}

To confirm the role of the GPI moiety in efficient insertion of the recombinant fluorescent protein into the membrane of target cells, purified GFP-GPI(DAF) was subjected to PI-PLC hydrolysis before the incubation with EL-4 cells. FACS analysis revealed efficient cell surface painting with intact, untreated GFP-GPI(DAF), whereas PI-PLC-cleaved GFP-GPI(DAF) failed to insert in the plasma membrane (ig. $5 A$ ). As a control, purified Thy-1 was added to PI-PLC treated GFP-GPI(DAF) after specific inactivation of the PI-PLC (33), revealing efficient painting of lymphocytes only with GPI-anchored Thy-1 (data not shown). These data confirmed the crucial role of the GPI anchor for efficient and stable membrane insertion.

We next investigated whether carrier lipids could influence the painting efficacy of GFP-GPI. To this end, purified GFP-GPI(DAF) was mixed with increasing concentrations of cholesterol, dipalmitoyl-phosphatidylethanolamine (DPPE) and sphingomyelin (SM). These lipids are known to be naturally enriched in membrane microdomains. Alternatively, GFP-GPI(DAF) was mixed with dioleoyl-phosphatidylethanolamine (DOPE), which is not enriched in lipid rafts (34). Liposomes made of lipids enriched in rafts increased the painting efficiency of GFP-GPI(DAF) in a concentration-dependent manner (Fig. $5 B$ ). Cholesterol at $0.6 \mu \mathrm{g} / \mathrm{ml}$ was the best carrier, giving a twofold increase in painting efficiency as assessed by FACS analysis. Both DPPE and SM enhanced the insertion of GFP-GPI(DAF) with optimal concentrations of 0.3 and 0.03 $\mu \mathrm{g} / \mathrm{ml}$, respectively. In contrast, addition of DOPE had no influence on cell surface painting (Fig. $\underline{5 B}$ ). Thus, the lipid environment is important for the efficiency of painting, and lipids that are naturally enriched in membrane microdomains facilitate the insertion of a GPI-anchored protein into lipid rafts.

\section{Exclusive localization of painted GFP-GPI(DAF) in lymphocyte lipid rafts}

To verify whether painted GFP-GPI fusion proteins also associated with lipid rafts, lymphocytes were incubated with purified GPI-anchored recombinant proteins for 2 and $36 \mathrm{~h}$ and washed, and the Triton X-100 lysates of painted cells were subjected to a sucrose density gradient fractionation. Both GFP-GPIs were found in the light density fractions corresponding to lipid rafts (Fig. 6). Interestingly, painted GFP-GPI(DAF) localized entirely in the raft fractions, 
similar to transfected GFP-GPI(DAF). However, a maximum of 55\% of GFP-GPI(TRAIL-R3) was found in microdomains. It has been reported that after painting GPI-anchored proteins reorganize in the plasma membrane and that their subsequent association with rafts may increase for up to $36 \mathrm{~h}$ after painting (38). In the case of our painted GFP-GPIs, this reorganization process apprears to be complete within $2 \mathrm{~h}$, since the same raft localization was observed in painted and stably transfected cells and painting for $36 \mathrm{~h}$ did not change the raft localization of either GFP-GPI (Fig. 6B). Endogenous proteins, such as acylated Fyn kinase, GPI-anchored Thy1 antigen, and the TCR/CD3 complex showed normal distribution at the plasma membrane in lymphocytes painted with either of the two GFP-GPI fusion proteins (Fig. 6A), suggesting that there is an intrinsic difference in the partitioning into lipid rafts of painted GFP-GPI(DAF) and GFP-GPI(TRAIL-R3). In conclusion, these experiments demonstrate that GPI-anchored GFPs can be stably inserted into the plasma membrane of a variety of cell types and that GFPGPI(DAF) serves as an ideal fluorescent marker for lipid rafts of live cells.

\section{DISCUSSION}

Lipid rafts are dynamic structures within the plasma membrane, and they play important roles in several aspects of cell physiology, including activation and sorting processes. They function as microdomain signaling centers, and a key property of lipid rafts lies in their ability to include or exclude certain proteins to a variable extent (3-6, 10, 11, 39). Indeed, upon specific triggering, transmembrane receptors such as the TCR, BCR, or TNFR1 translocate to lipid rafts, and this is a prerequisite for efficient receptor-mediated signal transduction $(3,35,40)$. However, the precise mechanism of the translocation to microdomains requires further investigation. Proteins with high lipid raft affinity are often GPI anchored, cholesterol linked, or palmitoylated/myristoylated $(1,5,6)$. Palmitoylation appears to increase lipid raft affinity but is not always sufficient for lipid raft association $(5,41)$. GPI-anchored proteins probably partition into lipid rafts as a result of preferential packing of their saturated lipid anchor (5, 7, 8, 42, 43).

The high affinity of GPI-anchored proteins for lipid raft localization encouraged us to generate recombinant GFP-GPIs as fluorescent marker for lipid rafts. In this present study, we have characterized two GFPs that have been inserted into the plasma membrane via distinct GPI anchors. The GFP inserted into the membrane via the GPI anchor derived from DAF was completely insoluble in $1 \%$ Triton $\mathrm{X}-100$ at $4^{\circ} \mathrm{C}$ and partitioned exclusively in lipid rafts. The raft association of GFP-GPI(TRAIL-R3) differed fundamentally, showing generally $<50 \%$ localization in microdomains. This precludes its use as a specific marker for rafts. Interestingly, Madore and et al. (21) also demonstrated that two other GPI-anchored proteins show different localization in neuronal plasma membranes: the prion protein $\operatorname{PrP}$ was found in high density primarily at the cell body and was relatively soluble in detergents. The Thy- 1 antigen was predominantly expressed at low density over the cell surface and was highly resistant to detergent solubilization (21). These findings are further substantiated by the fact that various GPI-anchored proteins partitioned to a different extent in lipid rafts of transfected CHO cells (44). These observations are crucial since GPI-anchored proteins have recently been used to study intracellular trafficking, and different cellular compartments have been visualized using GFP-GPIs $(12,15,19,43,45)$. In this respect, it is important to choose the right GPI coding sequence to target a GFP-GPI-fusion protein exclusively to lipid rafts. 
GPI-anchored proteins have the interesting property of integration into the plasma membrane of cells. This phenomenon was described some time ago when it was observed that purified DAF, when added to cells, inserted into the plasma membranes and was functional (46). Studies of humans harboring parasitic infections or studies on transgenic animals expressing DAF have provided evidence that membrane insertion of dissociated GPI-anchored proteins also occurs in vivo (47, 48). Exploiting this technique of cell surface painting has brought about a novel method for protein transfer (reviewed in ref 22). For example, cells painted with GPI-anchored MHC-peptide complexes became targets for killing by specific CTL (25), painting of tumor cells with GPI-anchored B7 was proposed as a novel tumor immune therapy approach (24), and painting of lymphocytes with KISS31-GPI allowed specific tumor homing (23). Cell surface painting offers several advantages over conventional transfection/infection methods: virtually any cell type can be painted, including primary cells that are difficult to transfect, and most proteins of interest can be expressed as GPI-anchored fusion proteins. The painting efficiency is high and reveals homogenous membrane insertion of the GPI-anchored protein. Painting is rapid as maximal insertion is achieved after 90 min to $2 \mathrm{~h}$, and protein exposure at the surface of the cell is immediate. Painting is performed under normal culture conditions with no detectable toxicity for the cells. Finally and most importantly, the introduced protein retains its biological function.

Detailed analysis by Premkumar et al. (38) of GPI anchors from many natural proteins revealed that painted GPI-anchored proteins can associate differently with membranes: either they may be incompletely or incorrectly inserted, or they may be inserted into the lipid bilayer as unassociated molecules. In our study, both painted GFP-GPI molecules were stably incorporated into plasma membrane microdomains. GFP-GPI(TRAIL-R3) partitioned to $\sim 20-50 \%$ in microdomains, whereas GFP-GPI(DAF) localized exclusively in lymphocyte lipid rafts. Interestingly, Premkumar et al. found that only $80 \%$ of native DAF and $21-46 \%$ of recombinant DAF after chase resided in lipid rafts. These differences in the membrane localization of DAF may be explained by the use of different ectodomains of these molecules. However, in our hands, changing the GFP molecule by the extracellular part of B7 did not alter the exclusive partitioning of the GPI-anchored molecule (data not shown). However, within the GPI-anchoring signal, the $\omega$ amino acid at the "cleavage-attachment site" and the amino acids $\omega+1$ and $\omega+2$ influence the transamidation efficiency $(49,50)$. Interestingly, DAF $(\omega=\mathrm{S}, \omega+1=\mathrm{G}$, and $\omega+2=\mathrm{T})$ has a high relative GPI-anchoring efficiency (50), whereas TRAIL-R3 $(\omega=\mathrm{A}, \omega+1=\mathrm{S}$, and $\omega+2=\mathrm{S})$ has a much lower relative GPI-anchoring efficiency. The C-terminal signal may also influence the localization of GPI-anchored molecules in the plasma membrane, where GPI-anchored proteins with a high relative GPI-anchoring efficiency predominantly localize in lipid rafts. The significant exclusion of GFP-GPI(TRAIL-R3) from lipid rafts may be due to the fine lipid composition of the GPI-anchor, to the palmitoylation of the inositol ring, to the amino acid sequence close to the GPI attachment site, or even to the fact that a significant proportion of the GFP is not modified by a GPI anchor and retains the hydrophobic amino acid tail. Although our data do not allow us to discriminate between these hypotheses, we herein show that exclusion from lipid rafts is affected neither by the removal of the TAPE repeats of TRAIL-R3 nor by the purification and painting process. Regarding GFP-GPI(DAF), painting of live lymphocytes depended entirely on the GPI anchor and was markedly enhanced in the presence of lipids that are naturally enriched in membrane microdomains, such as cholesterol, DPPE, and SM. These observations are in support of a recent study (51), showing increased spontaneous insertion of 
the GPI-anchored alkaline phosphatase into model lipid rafts in the presence of cholesterol. Thus, painting efficiency is increased in a lipid environment resembling lipid rafts.

Taken together, we have demonstrated that the source of a GPI anchor used to generate a GFPGPI as a fluorescent marker is critical for its membrane localization. In fact, GFP-GPI(DAF), but not GFP-GPI(TRAIL-R3), exclusively partition in lipid rafts. In addition, purified GFPGPI(DAF) could be stably inserted into lipid rafts of lymphocytes. Thus, painting with GFPGPI(DAF) will help to investigate the dynamic events in lipid rafts, such as formation of the immunological synapse, the reorganization of the signaling network at the synapse upon receptor engagement, as well as processes involved in protein sorting of live cells.

\section{ACKNOWLEDGMENTS}

We are grateful to Sandra Levrand for excellent technical assistance. We thank Dr. Caroline Johnson-Leger for critical reading and discussion of the manuscript. This study was supported by the Swiss National Science Foundation (SNSF 31-61960.00), the Giorgi-Cavalieri Foundation, and the German Research Foundation (DFG, TR SFB 11).

\section{REFERENCES}

1. Anderson, R. G., and Jacobson, K. (2002) A role for lipid shells in targeting proteins to caveolae, rafts, and other lipid domains. Science 296, 1821-1825

2. van Meer, G. (2002) Cell biology. The different hues of lipid rafts. Science 296, 855-857

3. Pierce, S. K. (2002) Lipid rafts and B-cell activation. Nat. Rev. Immunol. 2, 96-105

4. Edidin, M. (2001) Shrinking patches and slippery rafts: scales of domains in the plasma membrane. Trends Cell Biol. 11, 492-496

5. Simons, K., and Toomre, D. (2000) Lipid rafts and signal transduction. Nat. Rev. Mol. Cell Biol. 1, 31-39

6. Brown, D. A., and London, E. (2000) Structure and function of sphingolipid- and cholesterol-rich membrane rafts. J. Biol. Chem. 275, 17221-17224

7. Varma, R., and Mayor, S. (1998) GPI-anchored proteins are organized in submicron domains at the cell surface. Nature 394, 798-801

8. Friedrichson, T., and Kurzchalia, T. V. (1998) Microdomains of GPI-anchored proteins in living cells revealed by crosslinking. Nature 394, 802-805

9. Zacharias, D. A., Violin, J. D., Newton, A. C., and Tsien, R. Y. (2002) Partitioning of lipidmodified monomeric GFPs into membrane microdomains of live cells. Science 296, 913916

10. Sedwick, C. E., and Altman, A. (2002) Ordered just so: lipid rafts and lymphocyte function. SCi STKE 122, RE2 
11. Bromley, S. K., Burack, W. R., Johnson, K. G., Somersalo, K., Sims, T. N., Sumen, C., Davis, M. M., Shaw, A. S., Allen, P. M., and Dustin, M. L. (2001) The immunological synapse. Annu. Rev. Immunol. 19, 375-396

12. Nichols, B. J., Kenworthy, A. K., Polishchuk, R. S., Lodge, R., Roberts, T. H., Hirschberg, K., Phair, R. D., and Lippincott-Schwartz, J. (2001) Rapid cycling of lipid raft markers between the cell surface and Golgi complex. J. Cell Biol. 153, 529-541

13. Pelkmans, L., Kartenbeck, J., and Helenius, A. (2001) Caveolar endocytosis of simian virus 40 reveals a new two-step vesicular-transport pathway to the ER. Nat. Cell Biol. 3, 473-483

14. Lamaze, C., Dujeancourt, A., Baba, T., Lo, C. G., Benmerah, A., and Dautry-Varsat, A. (2001) Interleukin 2 receptors and detergent-resistant membrane domains define a clathrinindependent endocytic pathway. Mol. Cell 7, 661-671

15. Fivaz, M., Vilbois, F., Thurnheer, S., Pasquali, C., Abrami, L., Bickel, P. E., Parton, R. G., and van der Goot, F. G. (2002) Differential sorting and fate of endocytosed GPI-anchored proteins. EMBO J. 21, 3989-4000

16. Sabharanjak, S., Sharma, P., Parton, R. G., and Mayor, S. (2002) GPI-anchored proteins are delivered to recycling endosomes via a distinct cdc42-regulated, clathrin-independent pinocytic pathway. Dev. Cell 2, 411-423

17. Abrami, L., Liu, S., Cosson, P., Leppla, S. H., and Van Der Goot, F. G. (2003) Anthrax toxin triggers endocytosis of its receptor via a lipid raft-mediated clathrin-dependent process. J. Cell Biol. 160, 321-328

18. De Angelis, D. A., Miesenbock, G., Zemelman, B. V., and Rothman, J. E. (1998) PRIM: proximity imaging of green fluorescent protein-tagged polypeptides. Proc. Natl. Acad. Sci. USA 95, 12312-12316

19. Mayor, S., Sabharanjak, S., and Maxfield, F. R. (1998) Cholesterol-dependent retention of GPI-anchored proteins in endosomes. EMBO J. 17, 4626-4638

20. Magalhaes, A. C., Silva, J. A., Lee, K. S., Martins, V. R., Prado, V. F., Ferguson, S. S., Gomez, M. V., Brentani, R. R., and Prado, M. A. (2002) Endocytic intermediates involved with the intracellular trafficking of a fluorescent cellular prion protein. J. Biol. Chem. 277, 33311-33318

21. Madore, N., Smith, K. L., Graham, C. H., Jen, A., Brady, K., Hall, S., and Morris, R. (1999) Functionally different GPI proteins are organized in different domains on the neuronal surface. EMBO J. 18, 6917-6926

22. Medof, M. E., Nagarajan, S., and Tykocinski, M. L. (1996) Cell-surface engineering with GPI-anchored proteins. FASEB J. 10, 574-586

23. Legler, D. F., Johnson-Leger, C., Wiedle, G., Bron, C., and Imhof, B. A. (2004) The alpha v beta 3 integrin as a tumor homing ligand for lymphocytes. Eur. J. Immunol. 34, 1608-1616 
24. McHugh, R. S., Nagarajan, S., Wang, Y. C., Sell, K. W., and Selvaraj, P. (1999) Protein transfer of glycosyl-phosphatidylinositol-B7-1 into tumor cell membranes: a novel approach to tumor immunotherapy. Cancer Res. 59, 2433-2437

25. Huang, J. H., Getty, R. R., Chisari, F. V., Fowler, P., Greenspan, N. S., and Tykocinski, M. L. (1994) Protein transfer of preformed MHC-peptide complexes sensitizes target cells to T cell cytolysis. Immunity 1, 607-613

26. Zhang, F., Schmidt, W. G., Hou, Y., Williams, A. F., and Jacobson, K. (1992) Spontaneous incorporation of the glycosyl-phosphatidylinositol-linked protein Thy-1 into cell membranes. Proc. Natl. Acad. Sci. USA 89, 5231-5235

27. van den Berg, C. W., Cinek, T., Hallett, M. B., Horejsi, V., and Morgan, B. P. (1995) Exogenous glycosyl phosphatidylinositol-anchored CD59 associates with kinases in membrane clusters on U937 cells and becomes $\mathrm{Ca}(2+)$-signaling competent. J. Cell Biol. 131, 669-677

28. Walter, E. I., Ratnoff, W. D., Long, K. E., Kazura, J. W., and Medof, M. E. (1992) Effect of glycoinositolphospholipid anchor lipid groups on functional properties of decay-accelerating factor protein in cells. J. Biol. Chem. 267, 1245-1252

29. Luescher, B., and Bron, C. (1985) Biosynthesis of mouse Thy-1 antigen. J. Immunol. 134, 1084-1089

30. Schneider, P., Bodmer, J. L., Thome, M., Hofmann, K., Holler, N., and Tschopp, J. (1997) Characterization of two receptors for TRAIL. FEBS Lett. 416, 329-334

31. Sambrook, J. F., Fritsch, E. F., and Maniatis, T. (1989) Molecular Cloning: A Laboratory Manual, Cold Spring Harbor Laboratory Press, New York

32. Low, M. G., and Kincade, P. W. (1985) Phosphatidylinositol is the membrane-anchoring domain of the Thy-1 glycoprotein. Nature 318, 62-64

33. Mollby, R., and Wadstrom, T. (1973) Purification of phospholipase C (alpha-toxin) from Clostridium perfringens. Biochim. Biophys. Acta 321, 569-584

34. Legler, D. F., Doucey, M. A., Cerottini, J. C., Bron, C., and Luescher, I. F. (2001) Selective inhibition of CTL activation by a dipalmitoyl-phospholipid that prevents the recruitment of signaling molecules to lipid rafts. FASEB J. 15, 1601-1603

35. Legler, D. F., Micheau, O., Doucey, M. A., Tschopp, J., and Bron, C. (2003) Recruitment of TNF receptor 1 to lipid rafts is essential for TNFalpha-mediated NF-kappaB activation. Immunity 18, 655-664

36. Bordier, C. (1981) Phase separation of integral membrane proteins in Triton X-114 solution. J. Biol. Chem. 256, 1604-1607 
37. Nagarajan, S., Anderson, M., Ahmed, S. N., Sell, K. W., and Selvaraj, P. (1995) Purification and optimization of functional reconstitution on the surface of leukemic cell lines of GPIanchored Fc gamma receptor III. J. Immunol. Methods 184, 241-251

38. Premkumar, D. R., Fukuoka, Y., Sevlever, D., Brunschwig, E., Rosenberry, T. L., Tykocinski, M. L., and Medof, M. E. (2001) Properties of exogenously added GPI-anchored proteins following their incorporation into cells. J. Cell. Biochem. 82, 234-245

39. Horejsi, V. (2002) Membrane rafts in immunoreceptor signaling: new doubts, new proofs? Trends Immunol. 23, 562-564

40. Drevot, P., Langlet, C., Guo, X. J., Bernard, A. M., Colard, O., Chauvin, J. P., Lasserre, R., and He, H. T. (2002) TCR signal initiation machinery is pre-assembled and activated in a subset of membrane rafts. EMBO J. 21, 1899-1908

41. Melkonian, K. A., Ostermeyer, A. G., Chen, J. Z., Roth, M. G., and Brown, D. A. (1999) Role of lipid modifications in targeting proteins to detergent-resistant membrane rafts. Many raft proteins are acylated, while few are prenylated. J. Biol. Chem. 274, 3910-3917

42. Chatterjee, S., and Mayor, S. (2001) The GPI-anchor and protein sorting. Cell. Mol. Life Sci. 58, 1969-1987

43. Brown, D. A., and Rose, J. K. (1992) Sorting of GPI-anchored proteins to glycolipidenriched membrane subdomains during transport to the apical cell surface. Cell 68, 533-544

44. Cebecauer, M., Cerny, J., and Horejsi, V. (1998) Incorporation of leucocyte GPI-anchored proteins and protein tyrosine kinases into lipid-rich membrane domains of COS-7 cells. Biochem. Biophys. Res. Commun. 243, 706-710

45. Muniz, M., Morsomme, P., and Riezman, H. (2001) Protein sorting upon exit from the endoplasmic reticulum. Cell 104, 313-320

46. Medof, M. E., Kinoshita, T., and Nussenzweig, V. (1984) Inhibition of complement activation on the surface of cells after incorporation of decay-accelerating factor (DAF) into their membranes. J. Exp. Med. 160, 1558-1578

47. Rifkin, M. R., and Landsberger, F. R. (1990) Trypanosome variant surface glycoprotein transfer to target membranes: a model for the pathogenesis of trypanosomiasis. Proc. Natl. Acad. Sci. USA 87, 801-805

48. Kooyman, D. L., Byrne, G. W., McClellan, S., Nielsen, D., Tone, M., Waldmann, H., Coffman, T. M., McCurry, K. R., Platt, J. L., and Logan, J. S. (1995) In vivo transfer of GPI-linked complement restriction factors from erythrocytes to the endothelium. Science 269, 89-92

49. Gerber, L. D., Kodukula, K., and Udenfriend, S. (1992) Phosphatidylinositol glycan (PI-G) anchored membrane proteins. Amino acid requirements adjacent to the site of cleavage and PI-G attachment in the COOH-terminal signal peptide. J. Biol. Chem. 267, 12168-12173 
50. Chen, R., Knez, J. J., Merrick, W. C., and Medof, M. E. (2001) Comparative efficiencies of C-terminal signals of native glycophosphatidylinositol (GPI)-anchored proproteins in conferring GPI-anchoring. J. Cell. Biochem. 84, 68-83

51. Milhiet, P. E., Giocondi, M. C., Baghdadi, O., Ronzon, F., Roux, B., and Le Grimellec, C. (2002) Spontaneous insertion and partitioning of alkaline phosphatase into model lipid rafts. EMBO Rep. 3, 485-490

Received December 18, 2003; accepted September 15, 2004. 
Fig. 1
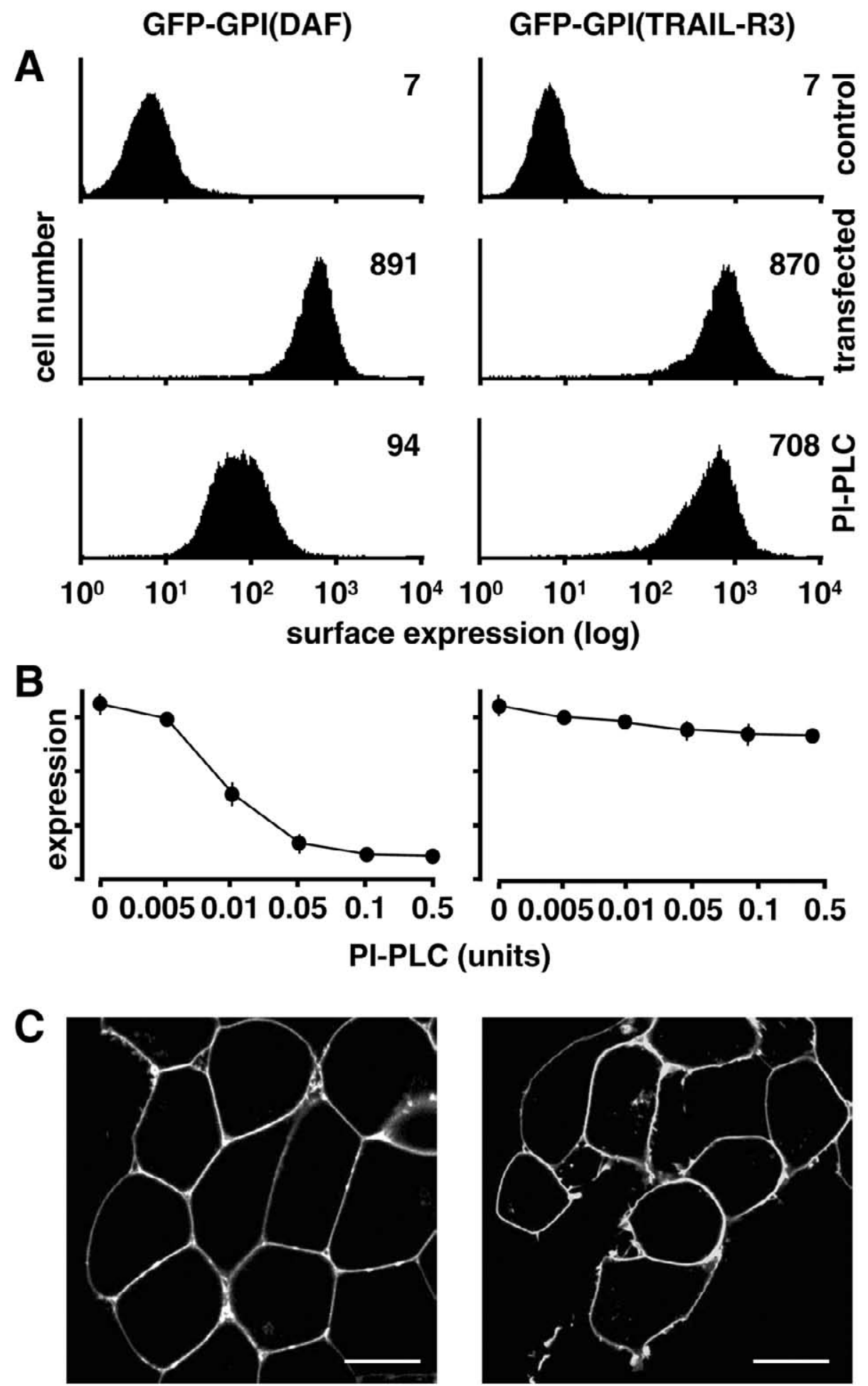

Figure 1. Both GPI-anchored GFPs are expressed at plasma membrane, but only GFP-GPI(DAF) is PI-PLC sensitive. A) Expression levels of GFP-GPI(DAF) and GFP-GPI(TRAIL-R3) of stable transfected HEK293 cells were analyzed by flow cytometry using untransfected cells as a control. Where indicated, transfected cells were incubated with 0.05 units of phosphatidylinositol-specific phospholipase C (PI-PLC). Numbers indicate mean fluorescent intensities from same experiment. B) Transfected cells were incubated for 90 min with increasing concentrations of PI-PLC, and surface expression of GFP-GPIs was analyzed by FACS analysis. $\boldsymbol{C}$ ) Cell surface expression of GFP-GPI(DAF) and GFPGPI(TRAIL-R3) on live HEK293 cells was analyzed by confocal microscopy. Bars $=10 \mu \mathrm{m}$. 
Fig. 2

\section{GFP-GPI(DAF)}

\section{GFP-GPI}

EGF R

Fyn,

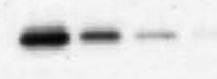

$-$

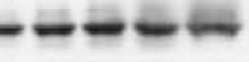

CTx

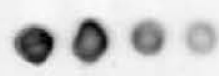

GFP-GPI(TRAIL-R3)
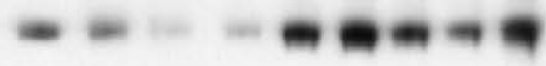

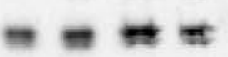

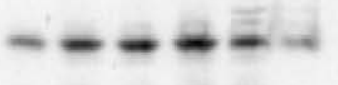

000

\section{kDa}
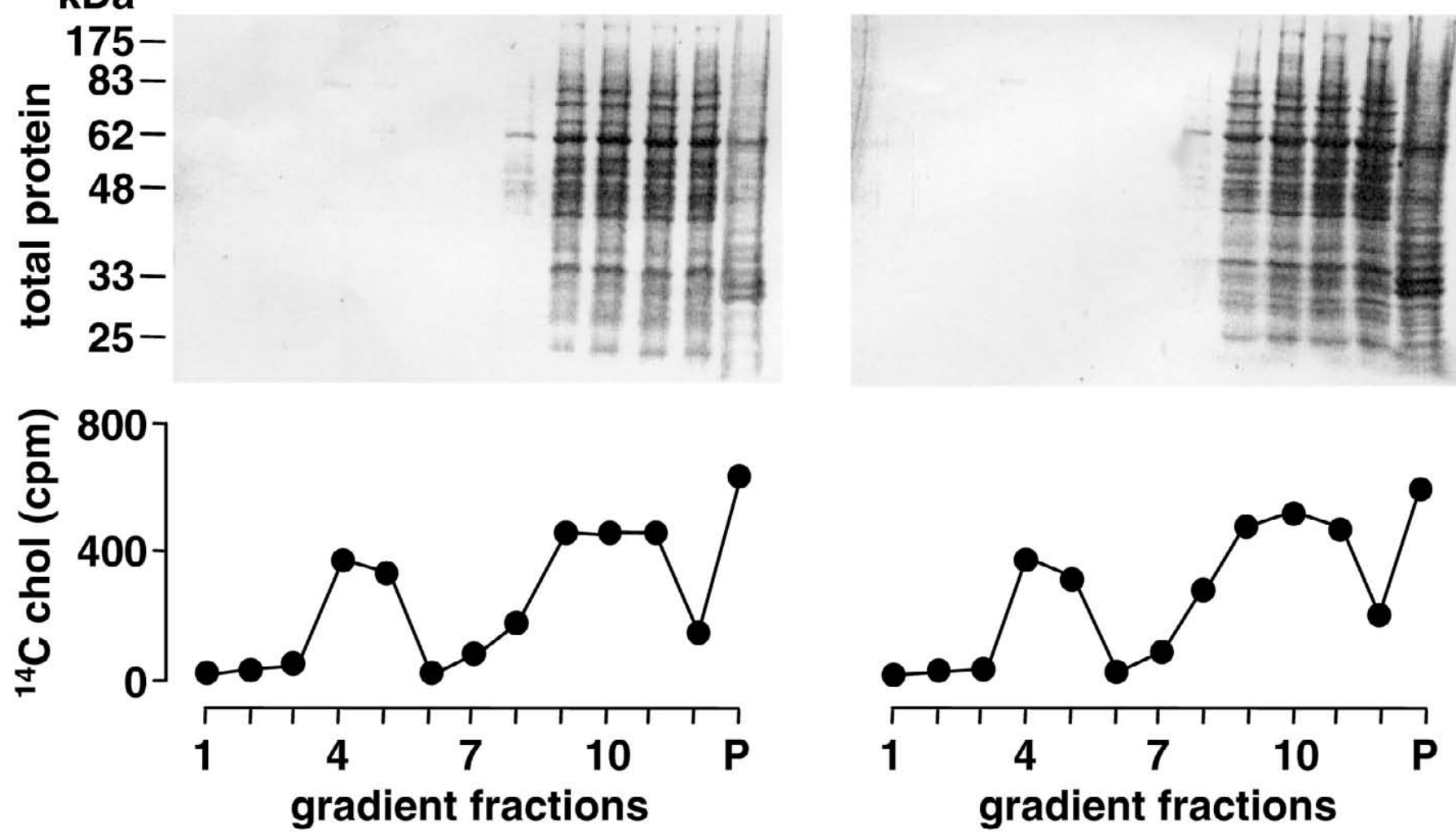

Figure 2. Different partitioning of GFP-GPI(DAF) and GFP-GPI(TRAIL-R3) in lipid rafts. HEK293 cells stably expressing GFP-GPI(DAF) and GFP-GPI(TRAIL-R3), respectively, were lysed in 1\% Triton X-100 and subjected to sucrose density gradient centrifugation. Proteins from equal volumes of collected representative fractions were separated by SDS-PAGE and analyzed by Western blotting using specific antibodies against GFP, EGF receptor, and Fyn. To analyze distribution of the ganglioside GM1, $5 \mu$ of each fraction were dot blotted onto a nitrocellulose membrane and detected by using $\mathrm{CTx}^{\mathrm{HRP}}$. Total protein distribution was monitored by Ponceau red staining. Additionally, cells were labeled with ${ }^{14} \mathrm{C}$ cholesterol before sucrose density gradient centrifugation and integrated counts were measured in each fraction. 
Fig. 3

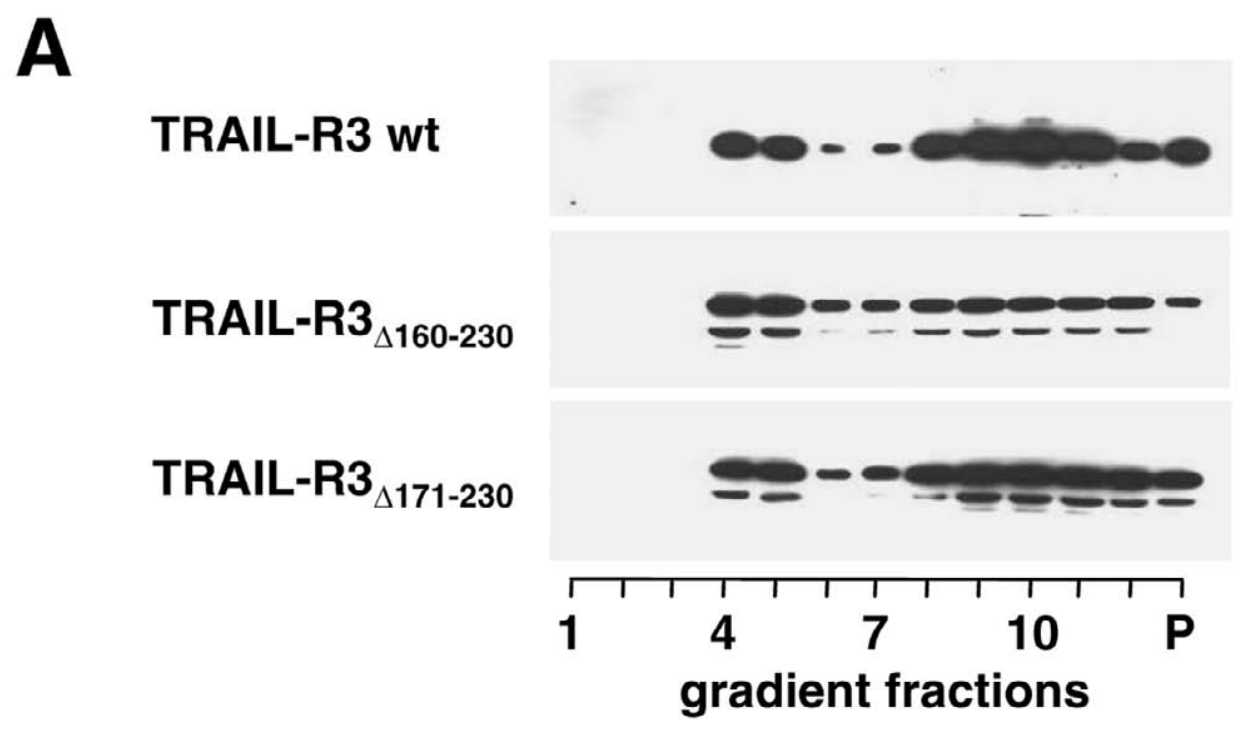

B

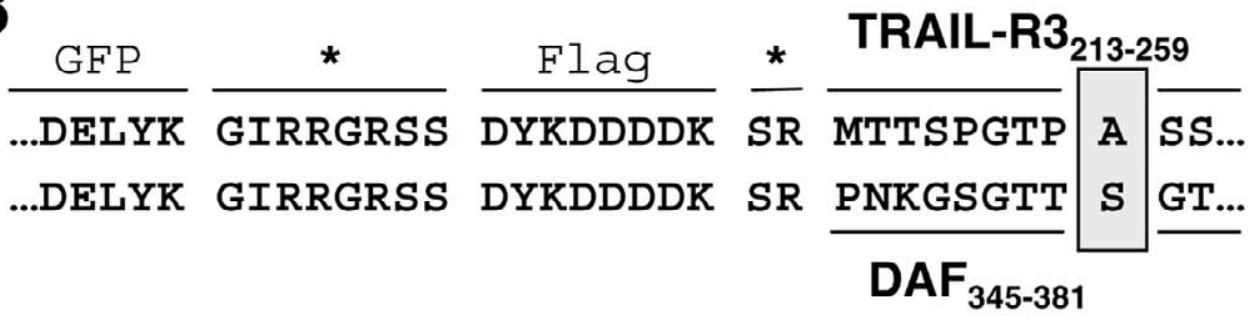

GFP-TRAIL-R3 $_{213-259}$

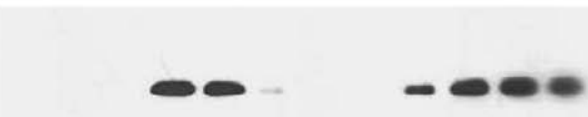

GFP-DAF $_{345-381}$

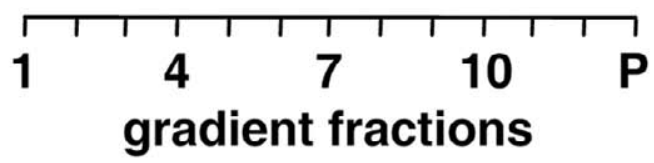

Figure 3. Structural analysis of GPI-anchored GFPs. $A$ ) HEK293 cells were transient transfected with Flag-tagged wild-type TRAIL-R3 (amino acids 1-259), Flag-tagged TRAIL-R3 lacking 4 out of 5 TAPE repeats (TRAIL-R3 ${ }_{\Delta 160-230}$ ), or Flag-tagged TRAIL-R3 lacking all 5 TAPE repeats (TRAIL-R3 ${ }_{\Delta 160-230}$ ). After $48 \mathrm{~h}$ of transfection, cells were lysed in $1 \%$ Triton X-100 and subjected to sucrose density gradient centrifugation to isolate lipid rafts. Proteins from equal volumes of collected representative fractions were separated by SDS-PAGE and analyzed by Western blotting using a specific antibody against Flag epitope. B) Amino acid sequence alignment and lipid raft localization of Flag-tagged GFP-

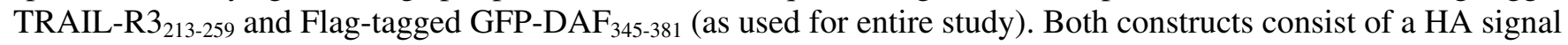
sequence fused to EGFP (GFP), followed by Flag epitope (Flag), and the last 9 amino acids of the mature protein preceding GPI anchor sequence (indicated by gray box). *Amino acids derived from restriction cloning sites. HEK293 cells were transfected with both constructs and subjected to lipid raft isolation. 
Fig. 4

\section{A GFP-GPI(DAF)}

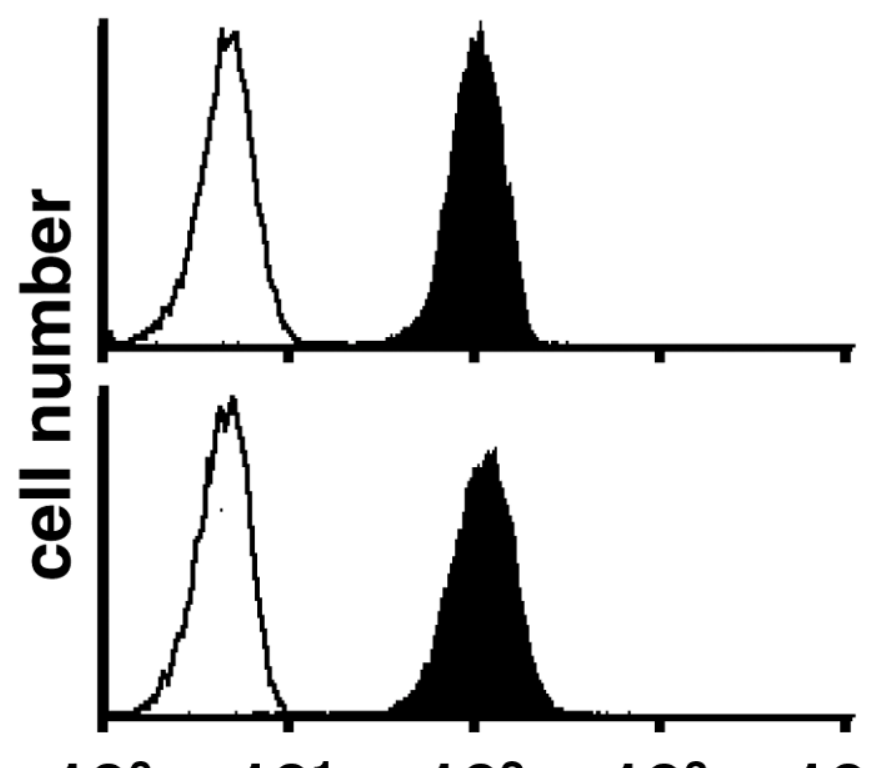

\section{GFP-GPI(TRAIL-R3)}
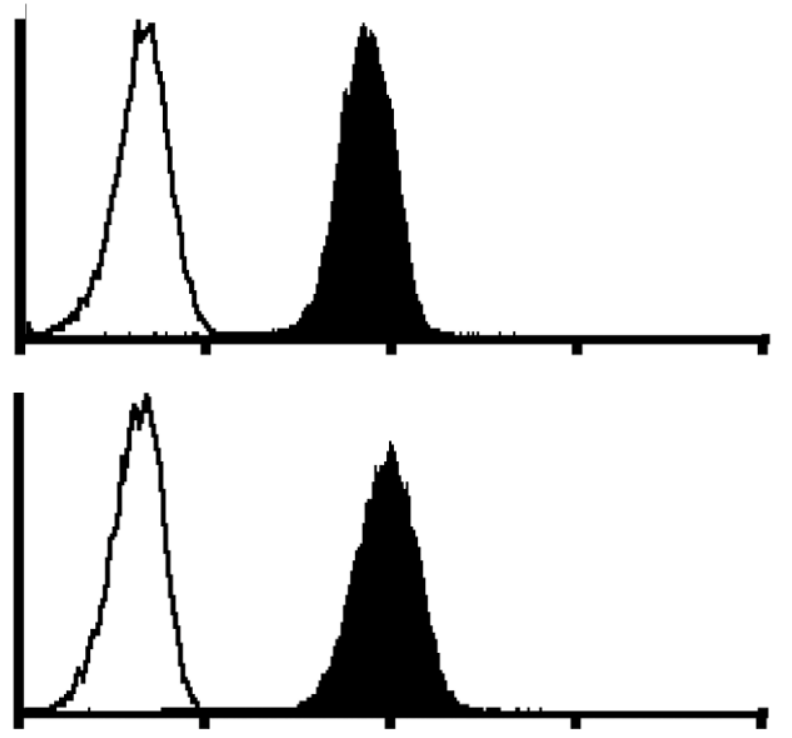

\section{$\overrightarrow{\mathbf{m}}$}

GFP-GPI(DAF)
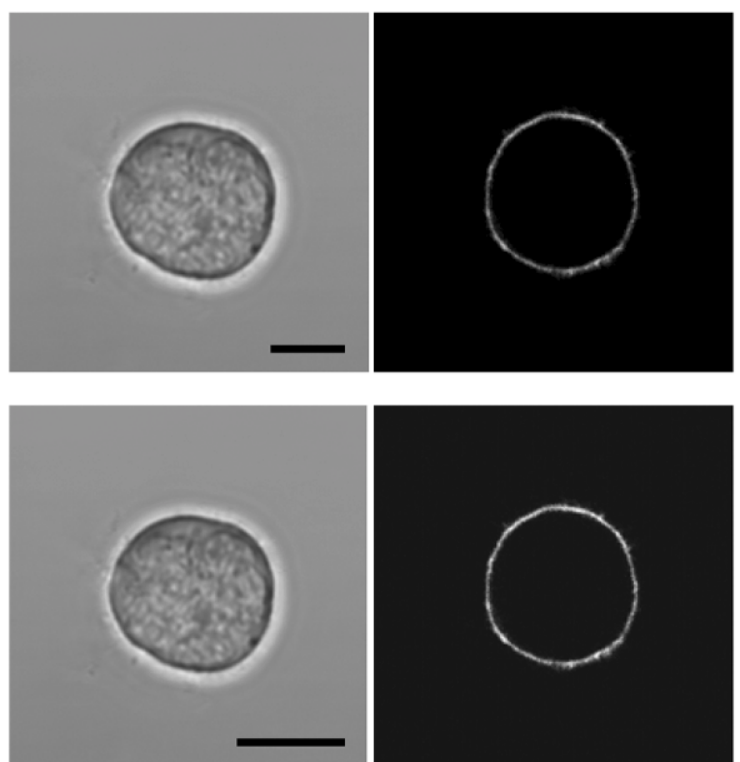
surface expression (log)

B

\section{GFP-GPI(TRAIL-R3)}
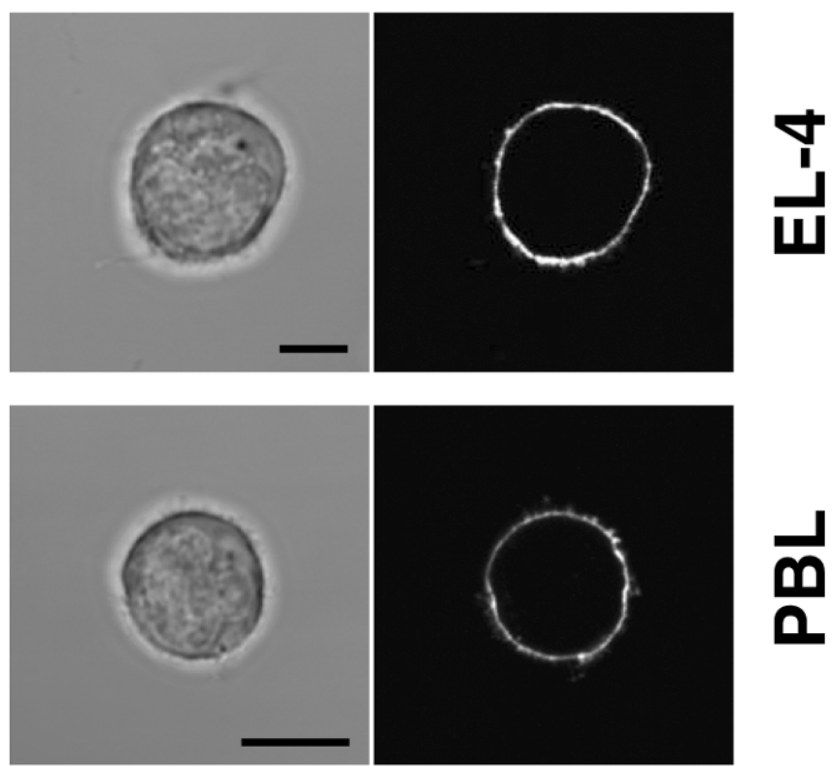

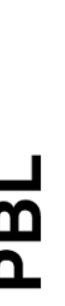
4 
Fig. 5
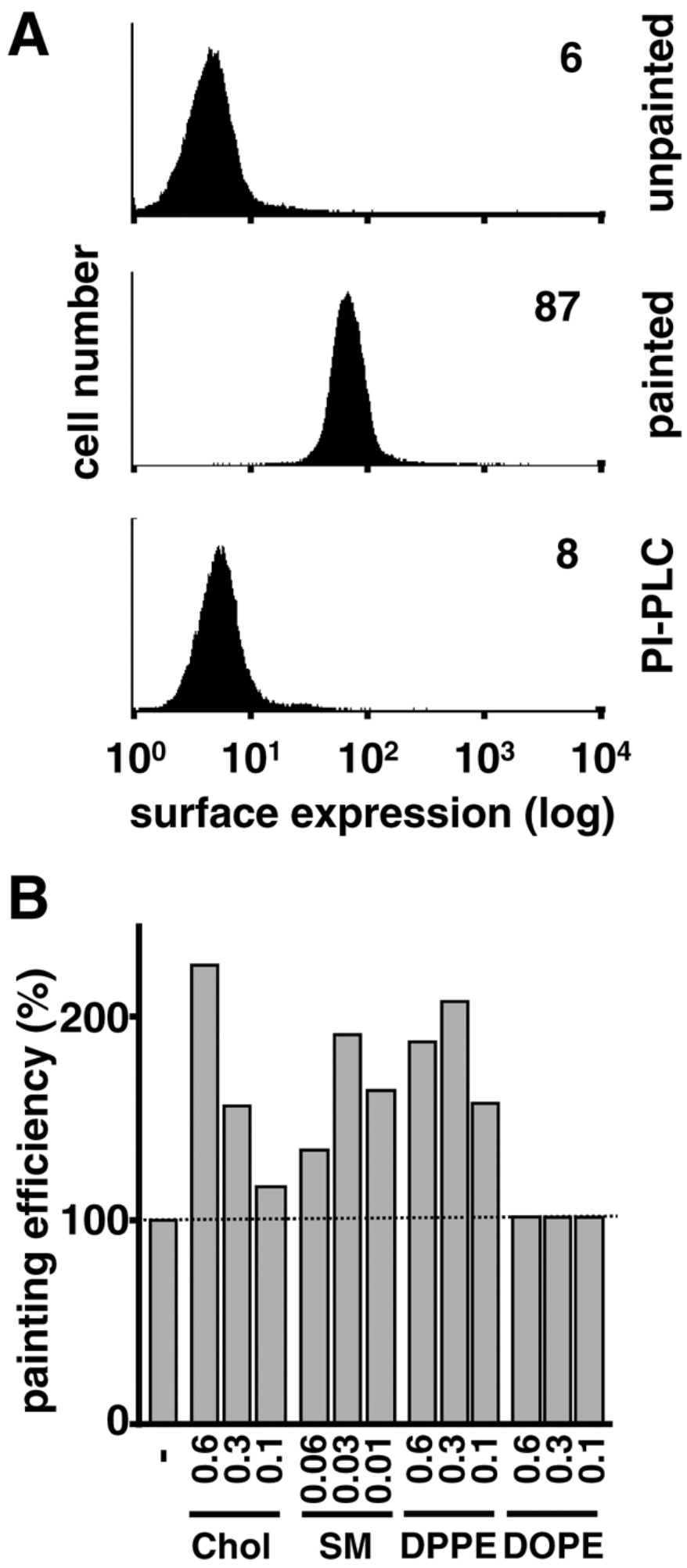

Figure 5. Painting with GFP-GPI(DAF) depends on GPI-anchor and painting efficiency can be increased by lipid environment. $A$ ) GPI-anchor of purified GFP-GPI(DAF) was cleaved or not by PI-PLC and then used for painting of EL4 cells, followed by FACS analysis. Mean fluorescent intensities are indicated. B) Liposomes made of 0.1-0.6 $\mu \mathrm{g} / \mathrm{ml}$ cholesterol (Chol), dipalmitoyl-phosphatidylethanolamine (DPPE), dioleoyl-phosphatidylethanolamine (DOPE), or 0.01$0.06 \mu \mathrm{g} / \mathrm{ml}$ sphingomyelin (SM) in the presence of purified GFP-GPI(DAF) was used for cell surface painting of EL-4 cells. Efficiency of membrane insertion of GFP-GPIs was analyzed by FACS. Mean value of fluorescent intensity of unpainted cells was 8 and 52 for GFP-GPI(DAF) painted cells in the absence of liposomes. 
Fig. 6

A

GFP-GPI(DAF)

Rafts

GFP-GPI

CD3E

Fyn

Thy-1

$-$
GFP-GPI(TRAIL-R3)

Rafts

Rarts
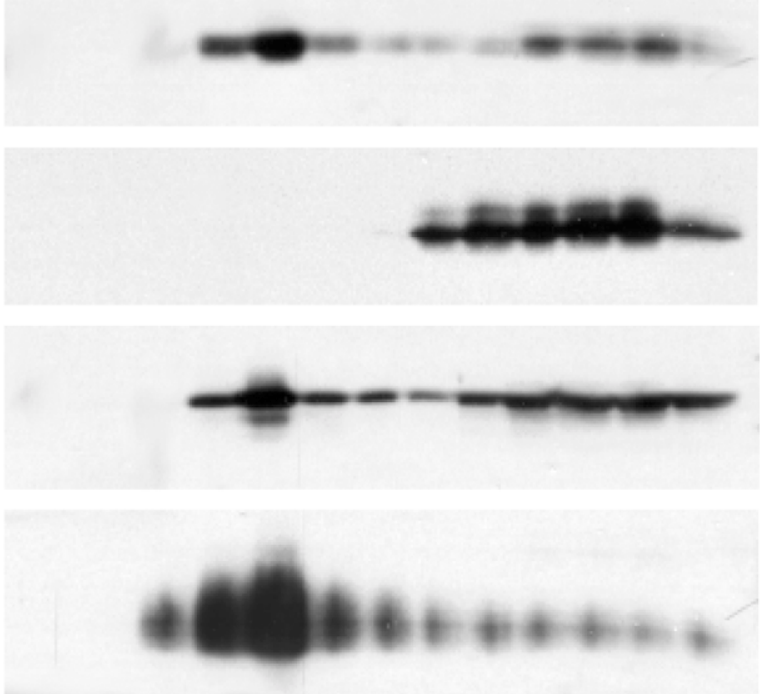

B

GFP-GPI

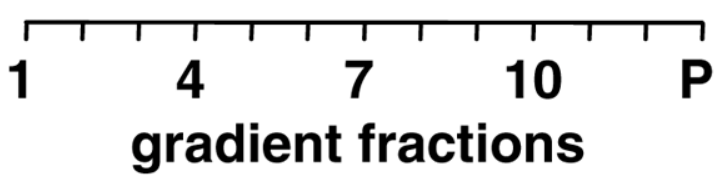

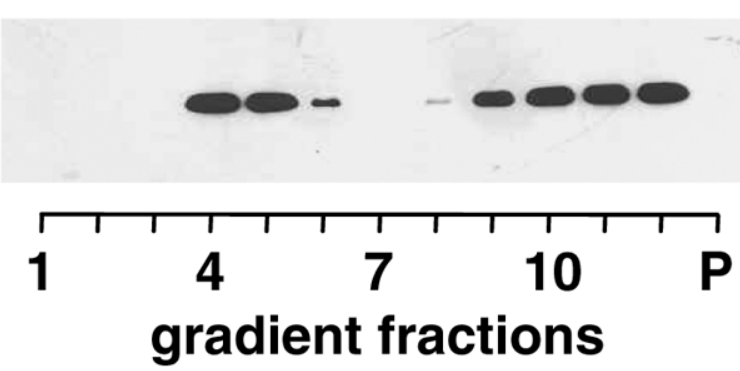

Figure 6. Cell surface painted GFP-GPI(DAF) exclusively localizes in lipid rafts. EL-4 T lymphocytes were painted separately with GFP-GPI(DAF) and GFP-GPI(TRAIL-R3) for $2 \mathrm{~h}(\boldsymbol{A})$ or $36 \mathrm{~h}(\boldsymbol{B})$ and washed extensively, and protein distribution of Triton X-100 cell lysates was analyzed by sucrose density gradient centrifugation. Proteins from equal volume of collected fractions were separated by SDS-PAGE and analyzed by Western blotting using specific antibodies against GFP, CD3e, Fyn, and Thy-1. 